\title{
Side jumps in the spin Hall effect: Construction of the Boltzmann collision integral
}

\author{
Dimitrie Culcer, ${ }^{1}$ E. M. Hankiewicz, ${ }^{2}$ Giovanni Vignale, ${ }^{3}$ and R. Winkler ${ }^{4,5}$ \\ ${ }^{1}$ Condensed Matter Theory Center, Department of Physics, University of Maryland, College Park, Maryland 20742-4111, USA \\ ${ }^{2}$ Institut für Theoretische Physik und Astrophysik, Universität Würzburg, 97074 Würzburg, Germany \\ ${ }^{3}$ Department of Physics and Astronomy, University of Missouri, Columbia, Missouri 65211, USA \\ ${ }^{4}$ Materials Science Division, Argonne National Laboratory, Argonne, Illinois 60439, USA \\ ${ }^{5}$ Northern Illinois University, De Kalb, Illinois 60115, USA
}

(Received 19 October 2009; published 29 March 2010)

\begin{abstract}
We present a systematic derivation of the side-jump contribution to the spin Hall current in systems without band-structure spin-orbit interactions, focusing on the construction of the collision integral for the Boltzmann equation. Starting from the quantum Liouville equation for the density operator we derive an equation describing the dynamics of the density matrix in the first Born approximation and to first order in the driving electric field. Elastic scattering requires conservation of the total energy, including the spin-orbit interaction energy with the electric field: this results in a first correction to the customary collision integral found in the Born approximation. A second correction is due to the change in the carrier position during collisions. It stems from the part of the density-matrix off-diagonal in wave vector. The two corrections to the collision integral add up and are responsible for the total side-jump contribution to the spin Hall current. The spin-orbit-induced correction to the velocity operator also contains terms diagonal and off-diagonal in momentum space, which together involve the total force acting on the system. This force is explicitly shown to vanish (on the average) in the steady state: thus the total contribution to the spin Hall current due to the additional terms in the velocity operator is zero.
\end{abstract}

DOI: 10.1103/PhysRevB.81.125332

\section{INTRODUCTION}

Semiconductor spin electronics is an active area of research in which both theory and experiment have made substantial progress during the past decade. ${ }^{1,2}$ The recent focus on electrically induced phenomena in systems with spin-orbit interactions has brought to light many unexplored and fascinating facets of semiconductor physics. Considerable progress has been made in past years in the electrical manipulation of spins in semiconductors, where experimental and theoretical work have yielded the prediction ${ }^{3-9}$ and discovery of the spin Hall effect. ${ }^{10-13}$ The spin Hall effect, ${ }^{1,8}$ which is the main focus of this paper, is the generation of a transverse spin current ${ }^{9,14}$ as a response to an external electric field. Such a spin current leads to a spin accumulation at the edges of the sample, and the relationship between spin currents and spin accumulation is nontrivial. ${ }^{15,16}$ The first observations of the spin Hall effect were followed by the measurement of the spin Hall effect at room temperature by optical techniques ${ }^{17}$ and the first successful measurement of the spin Hall effect in transport in ballistic $\mathrm{HgTe} / \mathrm{HgCdTe}$ quantum wells. ${ }^{18}$

Two main mechanisms have been shown to be responsible for the spin Hall effect. The presence of spin-orbit coupling in the impurity potentials gives rise to contributions to the spin Hall effect which are termed extrinsic. ${ }^{3-5,15,19-25}$ The spin Hall effect observed in Refs. 10 and 11 was shown by Engel et al.,${ }^{19}$ Tse and Das Sarma, ${ }^{20}$ and Hankiewicz and Vignale $^{23}$ to be due to extrinsic mechanisms. Band structure spin-orbit interactions yield contributions termed intrinsic. ${ }^{6,7,26-28}$ The spin Hall effect observed in Refs. 12 and 18 is believed to be due to intrinsic mechanisms. Extrinsic and intrinsic mechanisms were broadly discussed in the context of the anomalous Hall effect, which is the generation of a transverse charge and spin-polarization current in re-
PACS number(s): 72.25.Dc, 71.70.Ej, 85.75.-d

sponse to an electric field in a ferromagnetic medium. ${ }^{29-44}$ In fact, extrinsic contributions to the anomalous Hall and spin Hall effects are closely related. ${ }^{19,20}$ The interplay of intrinsic and extrinsic contributions is a complicated problem. It was first addressed by Tse and Das Sarma ${ }^{45}$ using an approach based on the diagrammatic Kubo formula. This was followed by a series of publications ${ }^{46-48}$ and this topic continues to be an active area of research. However, our focus in this work is on the case of extrinsic spin-orbit interactions alone and specifically the way they may be obtained from a kinetic equation approach.

Extrinsic contributions to the spin current are of two kinds. The first and more intuitive contribution arises from the asymmetric scattering of up and down spins known as skew scattering. This effect is found beyond the first Born approximation, i.e., provided one goes to at least third order in the electron-impurity potential. ${ }^{19,20,33}$ The effect appears naturally in the standard Boltzmann collision integral ${ }^{23,37}$ provided one goes beyond the first Born approximation. The associated spin Hall conductivity scales with disorder as the ordinary Drude conductivity ${ }^{19,20}$ (i.e., proportional to the electron-impurity scattering time), although it is of course much smaller.

The second extrinsic contribution has been known in the literature as side jump and has two main characteristics: (i) it appears already in the first Born approximation for the electron-impurity potential, and (ii) the associated spin Hall conductivity is independent of the electron-impurity scattering time-a surprising universality which will be fully explained below. In contrast to skew scattering, the side-jump mechanism cannot be derived straightforwardly from the standard form of the Boltzmann equation. Very early on, Luttinger $^{30}$ calculated the side-jump contribution to the charge conductivity using a recursive density-matrix approach, providing a thorough calculation yet leaving many 
questions unanswered concerning the physical picture behind different contributions. Later, Berger ${ }^{34,35}$ used a wave-packet formalism to build a semiclassical picture of side jump, identifying it with a shift of the center of mass of the wave packet during collisions. Nozières and Lewiner ${ }^{37}$ used this picture and carefully studied the side-jump contributions within a Boltzmann approach. These authors accounted for six contributions, some of which cancel each other; yet they did not associate any physical interpretation with the cancellations. In Ref. 15 the skew-scattering and side-jump terms were identified also within the framework of a drift-diffusion approach. In Ref. 23 it was claimed that the proper way to describe the side-jump effect is to replace the band energy in the usual Boltzmann collision integral by a modified band energy which includes corrections due to the spin-orbit interaction with the electric field. However, the validity of this claim has not been formally shown to date. Skew scattering and side jump were rigorously studied by Tse and Das Sarma ${ }^{20}$ using a diagrammatic Kubo formalism, demonstrating that the side-jump term can be identified with an anomalous current which gives rise to a renormalization of the current vertex. A derivation based on the Kubo formula was also presented in Ref. 49, finding that the side-jump contribution for the conduction band is independent of disorder and of the Coulomb potential to all orders in the strength of these interactions.

The purpose of this paper is to show a rigorous alternative derivation of the side jump within the framework of the kinetic equation, which does not require intuitive approaches and follows cleanly and clearly from the Boltzmann equation provided one constructs the collision integral with care. This construction, however, requires that we go beyond the Boltzmann equation formalism and resort to the full quantum Liouville equation for the one-particle density matrix, which we treat to second order in the electron-impurity potential and to first order in the electric field. In order to focus on the essential physics, we assume that band structure spin-orbit interactions are negligible. Thus we only take into account spin-orbit interactions with the impurities and with the external electric field that drives the current. In the present work, we first derive a general kinetic equation including the collision integral, then we solve the kinetic equation by separating the density matrix into equilibrium and nonequilibrium parts. Transport studies frequently perform these steps in the opposite order. Yet we find that our choice is more transparent for the present work.

Starting from the quantum Liouville equation we show rigorously that the conservation of energy in the Boltzmann collision integral must be modified to include the effective band energy, i.e., the bare band energy plus the spin-orbit interaction with the electric field. This modification is reflected in the appearance of a correction to the scattering term usually found in the Born approximation. This additional term, which is spin dependent and linear in the electric field, acts as a source for the spin current, yielding one half of the side-jump contribution.

The other half emerges when one takes into account the change in the position of the particle during collisions with impurities. The semiclassical picture of the renormalization of the trajectory of the electron during collisions will be de- rived rigorously from the scattering of electrons off the impurity potential which involves the terms off-diagonal in the wave vector in the density matrix. It turns out that this process and the modified conservation of energy give equal contributions to the spin Hall current, so that the full side-jump contribution can indeed be obtained by including twice the spin-orbit interaction energy with the electric field in the $\delta$ function of conservation of energy in the ordinary Boltzmann collision integral, as suggested previously in Ref. 23. However, the present derivation is rigorous and constitutes formal validation of this heuristic approach. We note that the factor of 2 emerges naturally from the diagrammatic Kubo formula, as demonstrated in Ref. 20, when one takes into account the vertex renormalization of the spin and charge currents.

In addition to constructing the picture of side jump outlined above, the analysis expounded in this work supports the argument that the full velocity operator contains the net force acting on the system, which must vanish on physical grounds. ${ }^{23}$ To provide a formal derivation of this fact, we show that the total contribution of the additional terms in the velocity operator to the spin Hall current is indeed zero. This is due to the fact that the velocity operator contains a spindependent term linear in the electric field, which reflects the spin-orbit interaction with the electric field, as well as an additional spin-dependent term off-diagonal in wave vector, which reflects the spin-orbit interaction with the impurities. The importance of this latter term was recognized already in diagrammatic linear-response theory in Ref. 20. Within the kinetic equation formalism, the two terms in the velocity operator produce equal and opposite contributions to the spin Hall current which cancel each other out. The physical interpretation of this fact is that, in the steady state, the average force acting on an electron must vanish.

In this paper, therefore, we strive to provide an understanding of the side-jump mechanism in the absence of intrinsic spin precession due to band-structure spin-orbit coupling, which is rigorous and at the same time physical. We believe that a rigorous physical understanding is a first step toward building a consistent picture, within the kinetic equation framework, of the interplay of spin precession due to band-structure spin-orbit coupling and spin-orbit coupling due to impurities. This interplay has been studied by Tse and Das Sarma ${ }^{45}$ based on a diagrammatic linear-response approach and by $\mathrm{Hu}$ et al. ${ }^{46}$ numerically. More recently Hankiewicz and Vignale ${ }^{47}$ constructed a phase diagram of this problem while Raimondi and $\mathrm{Schwab}^{48}$ employed a Keldysh Green's-function technique. The long-term aim of this work is to build a rigorous understanding, based on the kinetic equation, of intrinsic spin precession, skew scattering and side jump on an equal footing.

This article is organized as follows. In Sec. II a kinetic equation is derived starting from the quantum Liouville equation for the density operator. In Sec. III we explicitly construct the collision integral, including all contributions in the first Born approximation arising from the modification of the position operator. Section IV discusses the contributions of the side-jump mechanism to the spin Hall effect. It demonstrates that the corrections to the velocity operator do not contribute to the spin current. We end with conclusions. 


\section{TIME EVOLUTION OF THE DENSITY MATRIX}

We outline in this section the formalism that will be used to determine the collision integral and the way it gives rise to the side-jump spin Hall current. The system is described by a density operator $\hat{\rho}$, which obeys the quantum Liouville equation

$$
\frac{d \hat{\rho}}{d t}+\frac{i}{\hbar}\left[\hat{H}_{0}+\hat{H}_{E}+\hat{H}_{U}, \hat{\rho}\right]=0 .
$$

In this equation

$$
\hat{H}_{0}=\frac{\hbar^{2} \hat{k}^{2}}{2 m^{*}}
$$

is the Hamiltonian for a parabolic conduction band, with $m^{*}$ the carrier effective mass, while

$$
\begin{aligned}
\hat{H}_{E} & =e \boldsymbol{E} \cdot \hat{\boldsymbol{r}}+\lambda e \boldsymbol{E} \cdot \hat{\boldsymbol{\sigma}} \times \hat{\boldsymbol{k}} \\
& \equiv e \boldsymbol{E} \cdot \hat{\boldsymbol{r}}+\frac{1}{2} \hat{\boldsymbol{\sigma}} \cdot \hat{\boldsymbol{\Delta}}_{k}
\end{aligned}
$$

represents the interaction with a constant uniform external electric field $\boldsymbol{E}$. The term $\hat{H}_{E}$ includes both the direct interaction and the interaction via the (material-dependent) spinorbit coupling of strength $\lambda$. We have used the notation $\hat{\boldsymbol{\Delta}}_{\boldsymbol{k}}$ $=2 \lambda e \hat{\boldsymbol{k}} \times \boldsymbol{E}$ for the effective field characterizing the spindependent part of this interaction, a term which, as we shall see later, gives rise to one half of the side-jump contribution to the spin Hall conductivity. Finally

$$
\hat{H}_{U}=U(\hat{\boldsymbol{r}})+\lambda \nabla U(\hat{\boldsymbol{r}}) \cdot \hat{\boldsymbol{\sigma}} \times \hat{\boldsymbol{k}}
$$

denotes the interaction with a set of randomly distributed impurities, again both directly and via spin-orbit coupling. In order for the scalar part of the potential to exceed its spindependent part we require $\lambda k_{F}^{2} \ll 1$.

We project the Liouville equation onto a set of timeindependent states $\{|\boldsymbol{k} s\rangle\}$ of definite wave vector $\boldsymbol{k}$ and spin orientation $s= \pm 1$ along the $z$ axis. The matrix elements of $\hat{\rho}$ in this basis form the spin-density matrix and are written as $\rho_{\boldsymbol{k} \boldsymbol{k}^{\prime}} \equiv \rho_{\boldsymbol{k} \boldsymbol{k}^{\prime}}^{s s^{\prime}}=\left\langle\boldsymbol{k} s|\hat{\rho}| \boldsymbol{k}^{\prime} s^{\prime}\right\rangle$ with corresponding notations for the matrix elements of $\hat{H}_{0}, \hat{H}_{E}$, and $\hat{H}_{U}$. We assume that impurities are uncorrelated and the normalization is such that the configurational average of $\left\langle\boldsymbol{k} s\left|\hat{H}_{U}\right| \boldsymbol{k}^{\prime} s^{\prime}\right\rangle\left\langle\boldsymbol{k}^{\prime} s^{\prime}\left|\hat{H}_{U}\right| \boldsymbol{k} s\right\rangle$ is $\left(n_{i} / V\right)\left|\bar{U}_{\boldsymbol{k} k^{\prime}}\right|^{2} \delta_{s s^{\prime}}$, where $n_{i}$ is the impurity density, $V$ the crystal volume, and

$$
\bar{U}_{\boldsymbol{k} \boldsymbol{k}^{\prime}}=\mathcal{U}_{\boldsymbol{k} \boldsymbol{k}^{\prime}}\left(1-i \lambda \boldsymbol{\sigma} \cdot \boldsymbol{k} \times \boldsymbol{k}^{\prime}\right) .
$$

Here $\mathcal{U}_{\boldsymbol{k} \boldsymbol{k}^{\prime}}$ are the matrix elements of the electron-impurity potential $U(\hat{\boldsymbol{r}})$ between plane waves while $\bar{U}_{\boldsymbol{k} \boldsymbol{k}^{\prime}}$ is reserved for the total potential of a single impurity including the spinorbit contribution. In what follows spin indices will be suppressed and all quantities are assumed to be matrices in spin space. Note that we use the convention that $\bar{U}_{\boldsymbol{k} \boldsymbol{k}^{\prime}}$ and $\mathcal{U}_{\boldsymbol{k} \boldsymbol{k}^{\prime}}$ have units of energy $\times$ volume.
The density matrix $\hat{\rho}$ is divided into a part diagonal in $\boldsymbol{k}$ and a part off-diagonal in $\boldsymbol{k}$, given by $\rho_{\boldsymbol{k} \boldsymbol{k}^{\prime}}=f_{\boldsymbol{k}} \delta_{\boldsymbol{k} \boldsymbol{k}^{\prime}}+g_{\boldsymbol{k} \boldsymbol{k}^{\prime}}$. These two parts of $\hat{\rho}$ satisfy a set of coupled equations

$$
\begin{gathered}
\frac{d f_{k}}{d t}+\frac{i}{\hbar}[\hat{H}, \hat{f}]_{k k}=-\frac{i}{\hbar}\left[\hat{H}_{U}, \hat{g}\right]_{k k} \\
\frac{d g_{k k^{\prime}}}{d t}+\frac{i}{\hbar}[\hat{H}, \hat{g}]_{k k^{\prime}}=-\frac{i}{\hbar}[\hat{H} \\
U, \hat{f}]_{k k^{\prime}}-\frac{i}{\hbar}\left[\hat{H}_{U}, \hat{g}\right]_{k k^{\prime}},
\end{gathered}
$$

where $\hat{H} \equiv \hat{H}_{0}+\hat{H}_{E}$. In the first Born approximation the solution to Eq. (6b) for $g_{k k^{\prime}}$ is

$g_{\boldsymbol{k} \boldsymbol{k}^{\prime}}=-\left.\frac{i}{\hbar} \lim _{\eta \rightarrow 0} \int_{0}^{\infty} d t^{\prime} e^{-\eta t^{\prime}} e^{-i \hat{H} t^{\prime} / \hbar}\left[\hat{H}_{U}, \hat{f}\left(t-t^{\prime}\right)\right] e^{i \hat{H} t^{\prime} / \hbar}\right|_{k k^{\prime}}$,

where $\eta>0$ is a regularization factor. We are considering variations which are slow on the scale of the momentum relaxation time, thus we approximate $\hat{f}\left(t-t^{\prime}\right)$ in the integral by $\hat{f}(t),{ }^{50}$ which is written simply as $\hat{f}$, and satisfies the equation

$$
\frac{d f_{k}}{d t}+\frac{i}{\hbar}[\hat{H}, \hat{f}]_{k k}+\hat{J}\left(f_{k}\right)=0,
$$

where the scattering term $\hat{J}\left(f_{k}\right)$ is

$$
\begin{aligned}
\hat{J}\left(f_{k}\right) & =(i / \hbar)\left[\hat{H}_{U}, \hat{g}\right]_{k k} \\
& =\left.\frac{1}{\hbar^{2}} \lim _{\eta \rightarrow 0} \int_{0}^{\infty} d t^{\prime} e^{-\eta t^{\prime}}\left[\hat{H}_{U}, e^{-i \hat{H} t^{\prime} / \hbar}\left[\hat{H}_{U}, \hat{f}\right] e^{i \hat{H} t^{\prime} / \hbar}\right]\right|_{k k} .
\end{aligned}
$$

Equations (8) and (9) describe the dynamics of the density matrix and constitute the complete set of tools we require in order to derive the kinetic equation satisfied by the density matrix in an electric field, including the side-jump contribution to the scattering term due to the modification of the position operator by the spin-orbit interaction.

\section{DERIVATION OF THE COLLISION INTEGRAL}

We want to evaluate further the collision integral (9). For this purpose, we decompose the matrix $f_{k}$ into a part scalar in spin space and a spin-dependent part, thus $f_{k}=n_{k} \rrbracket+S_{k}$, with $\rrbracket$ the identity matrix and $S_{k}$ expressible in terms of the Pauli matrices. We will show in the following that, to first order in $\lambda$ and in the electric field, we can write the scattering term as

$$
\hat{J}\left(f_{k}\right)=\hat{J}_{0}\left(n_{k}\right)+\hat{J}_{\mathrm{sj}}^{a}\left(n_{k}\right)+\hat{J}_{\mathrm{sj}}^{b}\left(n_{k}\right)+\hat{J}_{0}\left(S_{k}\right) .
$$

The first of these terms comes from the band Hamiltonian $\hat{H}_{0}$ is a scalar in spin space and does not depend on $\lambda$ or on the electric field. The second term reflects the fact that the total energy must be conserved during scattering events, including the second term in Eq. (3). The third term comes from the direct interaction with the electric field $e \boldsymbol{E} \cdot \hat{\boldsymbol{r}}$ and arises because $\boldsymbol{r}$ fails to commute with the spin-orbit interaction with the impurities. Physically this reflects the change in $\boldsymbol{r}$ during a collision with an impurity. The resulting variation in $e \boldsymbol{E} \cdot \boldsymbol{r}$ also contributes to the overall energy balance. Both $\hat{J}_{\mathrm{sj}}^{a}\left(n_{\boldsymbol{k}}\right)$ 
and $\hat{J}_{\mathrm{sj}}^{b}\left(n_{k}\right)$ are spin dependent and are linear in $\lambda$ and the electric field $\boldsymbol{E}$. We will discuss each of the contributions in turn below. The last scattering term, $\hat{J}_{0}\left(S_{k}\right)$, will be important in the kinetic equation below in determining the steady-state correction linear in $\boldsymbol{E}$ to the spin-dependent part of the density matrix, and thus to the spin Hall current.

\section{A. Scattering correction due to the conservation of the modified carrier energy}

In Sec. III A we focus on the part of the scattering term which is linear in the electric field and arises from the addi- tion of the spin-orbit interaction with the electric field $\frac{1}{2} \boldsymbol{\sigma} \cdot \boldsymbol{\Delta}_{\boldsymbol{k}}$ to the particle energy $\varepsilon_{0 k} \equiv \hbar^{2} k^{2} /\left(2 m^{*}\right)$. Since we are working to first order in $\lambda$ and in the electric field, in Sec. III A we only need to consider the scalar part of the impurity potential, $\mathcal{U}_{\boldsymbol{k} \boldsymbol{k}^{\prime}}$. Moreover the time evolution operators in Sec. III A include the side-jump energy $\frac{1}{2} \boldsymbol{\sigma} \cdot \boldsymbol{\Delta}_{\boldsymbol{k}}$, but not the term $e \boldsymbol{E} \cdot \boldsymbol{r}$, which will be considered in Sec. III B. The matrix elements of $\hat{H}$ in the exponents of the time-evolution operators are thus diagonal in $\boldsymbol{k}$.

Writing out all the terms in the double commutator, Eq. (9b), we find

$$
\begin{aligned}
& \frac{1}{\hbar^{2}}\left[\hat{H}_{U}, e^{-i \hat{H} t^{\prime} / \hbar}\left[\hat{H}_{U}, \hat{f}\right] e^{i \hat{H} t^{\prime} / \hbar}\right]_{k \boldsymbol{k}}=\frac{n_{i}}{\hbar^{2}} \int \frac{d^{d} k^{\prime}}{(2 \pi)^{d}}\left(\mathcal{U}_{\boldsymbol{k} \boldsymbol{k}^{\prime}} e^{-i H_{\boldsymbol{k}^{\prime}} t^{\prime} / \hbar} \mathcal{U}_{\boldsymbol{k}^{\prime} k} f_{\boldsymbol{k}} e^{i H_{\boldsymbol{k}} t^{\prime} / \hbar}-\mathcal{U}_{\boldsymbol{k} \boldsymbol{k}^{\prime}} e^{-i H_{\boldsymbol{k}^{\prime}} t^{\prime} / \hbar} f_{\boldsymbol{k}^{\prime}} \mathcal{U}_{\boldsymbol{k}^{\prime} \boldsymbol{k}} e^{i H_{\boldsymbol{k}^{t^{\prime}}} / \hbar}\right. \\
& \left.-e^{-i H_{\boldsymbol{k}^{\prime}}{ }^{\prime} / \hbar} \mathcal{U}_{\boldsymbol{k} \boldsymbol{k}^{\prime}} f_{\boldsymbol{k}^{\prime}} e^{i H_{\boldsymbol{k}^{\prime}} t^{\prime} / \hbar} \mathcal{U}_{\boldsymbol{k}^{\prime} \boldsymbol{k}}+e^{-i H_{\boldsymbol{k}^{\prime}} t^{\prime} / \hbar} f_{\boldsymbol{k}} \mathcal{U}_{\boldsymbol{k} \boldsymbol{k}^{\prime}} e^{i H_{\boldsymbol{k}^{\prime} t^{\prime}} / \hbar} \mathcal{U}_{\boldsymbol{k}^{\prime} \boldsymbol{k}}\right) \text {, }
\end{aligned}
$$

where $H_{k}=\varepsilon_{0 k}+\frac{1}{2} \boldsymbol{\sigma} \cdot \boldsymbol{\Delta}_{k}$ and $d$ is the dimensionality of the system. By expanding the exponentials of Pauli matrices, the product of time-evolution operators can be written, to first order in $\lambda$, as

$$
\begin{aligned}
e^{-i H_{\boldsymbol{k}^{\prime}} t^{\prime} / \hbar} e^{i H_{k} t^{\prime} / \hbar}= & e^{i\left(\varepsilon_{0 k^{-}}-\varepsilon_{0 \boldsymbol{k}^{\prime}}\right) t^{\prime} / \hbar}\left[\cos \frac{\Delta_{\boldsymbol{k}} t^{\prime}}{2 \hbar} \cos \frac{\Delta_{k}^{\prime} t^{\prime}}{2 \hbar}\right. \\
& -i \boldsymbol{\sigma} \cdot \hat{\boldsymbol{\Delta}}_{\boldsymbol{k}^{\prime}} \cos \frac{\Delta_{\boldsymbol{k}} t^{\prime}}{2 \hbar} \sin \frac{\Delta_{\boldsymbol{k}}^{\prime} t^{\prime}}{2 \hbar} \\
& \left.+i \boldsymbol{\sigma} \cdot \hat{\boldsymbol{\Delta}}_{\boldsymbol{k}} \sin \frac{\Delta_{k} t^{\prime}}{2 \hbar} \cos \frac{\Delta_{k}^{\prime} t^{\prime}}{2 \hbar}\right],
\end{aligned}
$$

where $\hat{\boldsymbol{\Delta}}_{\boldsymbol{k}}$ is a unit vector in $\boldsymbol{\Delta}_{\boldsymbol{k}}$ direction.

The only task that remains is integration over the time variable $t^{\prime}$, giving a series of $\delta$ functions reflecting energy conservation. The overall result for this scattering term, to first order in $\lambda$, can be decomposed into a scalar part $\hat{J}_{0}\left(n_{k}\right)$ independent of $\lambda$, and spin-dependent parts $\hat{J}_{\mathrm{sj}}^{a}\left(n_{k}\right)+\hat{J}_{0}\left(S_{k}\right)$. These parts may be written as follows:

$$
\begin{aligned}
\hat{J}_{0}\left(n_{k}\right)= & \frac{\pi n_{i}}{2 \hbar} \int \frac{d^{d} k^{\prime}}{(2 \pi)^{d}}\left|\mathcal{U}_{\boldsymbol{k} k^{\prime}}\right|^{2}\left(n_{k}-n_{\boldsymbol{k}^{\prime}}\right)\left[\delta\left(\epsilon_{+}-\epsilon_{+}^{\prime}\right)+\delta\left(\epsilon_{-}-\epsilon_{-}^{\prime}\right)\right. \\
& \left.+\delta\left(\epsilon_{+}-\epsilon_{-}^{\prime}\right)+\delta\left(\epsilon_{-}-\epsilon_{+}^{\prime}\right)\right] \\
\hat{J}_{\mathrm{sj}}^{a}\left(n_{\boldsymbol{k}}\right)= & \frac{\pi n_{i}}{2 \hbar} \int \frac{d^{d} k^{\prime}}{(2 \pi)^{d}}\left|\mathcal{U}_{\boldsymbol{k} k^{\prime}}\right|^{2}\left(n_{k}-n_{\boldsymbol{k}^{\prime}}\right)\left\{\boldsymbol{\sigma} \cdot\left(\hat{\boldsymbol{\Delta}}_{\boldsymbol{k}}+\hat{\boldsymbol{\Delta}}_{\boldsymbol{k}^{\prime}}\right)\right. \\
& \times\left[\delta\left(\epsilon_{+}-\epsilon_{+}^{\prime}\right)-\delta\left(\epsilon_{-}-\epsilon_{-}^{\prime}\right)\right] \\
& \left.+\boldsymbol{\sigma} \cdot\left(\hat{\boldsymbol{\Delta}}_{k}-\hat{\boldsymbol{\Delta}}_{\boldsymbol{k}^{\prime}}\right)\left[\delta\left(\epsilon_{+}-\epsilon_{-}^{\prime}\right)-\delta\left(\epsilon_{-}-\epsilon_{+}^{\prime}\right)\right]\right\},
\end{aligned}
$$

$$
\hat{J}_{0}\left(S_{\boldsymbol{k}}\right)=\frac{2 \pi n_{i}}{\hbar} \int \frac{d^{d} k^{\prime}}{(2 \pi)^{d}}\left|\mathcal{U}_{\boldsymbol{k} \boldsymbol{k}^{\prime}}\right|^{2}\left(S_{\boldsymbol{k}}-S_{\boldsymbol{k}^{\prime}}\right) \delta\left(\varepsilon_{0 \boldsymbol{k}}-\varepsilon_{0 \boldsymbol{k}^{\prime}}\right) .
$$

The full energies $\epsilon_{ \pm}=\epsilon_{0 k} \pm \Delta_{k} / 2$ and $\epsilon_{ \pm}^{\prime}=\varepsilon_{0 k^{\prime}} \pm \Delta_{k^{\prime}} / 2$, where $\Delta_{k}=\left|\Delta_{k}\right|$. The scalar term $\hat{J}_{0}\left(n_{k}\right)$ reproduces the ordinary Boltzmann-equation scattering term. The side-jump term $\hat{J}_{\mathrm{sj}}^{a}\left(n_{k}\right)$ constitutes a correction that reflects the presence of the spin-orbit interaction energy with the electric field in the condition for energy conservation. We expand the $\delta$ functions in this scattering term in $\Delta_{k}$ as

$$
\delta\left(\epsilon_{+}-\epsilon_{+}^{\prime}\right)=\delta\left(\varepsilon_{0 k}-\varepsilon_{0 k^{\prime}}\right)+\left(\frac{\Delta_{k}}{2}-\frac{\Delta_{k^{\prime}}}{2}\right) \frac{\partial}{\partial \varepsilon_{0 k}} \delta\left(\varepsilon_{0 k}-\varepsilon_{0 k^{\prime}}\right)
$$

with corresponding expressions for the other combinations of $\delta$ functions. Adding all contributions together the scattering term $\hat{J}_{\mathrm{sj}}^{a}\left(n_{k}\right)$ simplifies considerably and we obtain the final expression

$$
\begin{aligned}
\hat{J}_{\mathrm{sj}}^{a}\left(n_{k}\right)= & \frac{2 \pi n_{i}}{\hbar} \int \frac{d^{d} k^{\prime}}{(2 \pi)^{d}}\left|\mathcal{U}_{k k^{\prime}}\right|^{2}\left(n_{k}-n_{k^{\prime}}\right) \frac{1}{2} \boldsymbol{\sigma} \cdot\left(\boldsymbol{\Delta}_{k}-\boldsymbol{\Delta}_{k^{\prime}}\right) \\
& \times \frac{\partial}{\partial \varepsilon_{0 k}} \delta\left(\varepsilon_{0 k}-\varepsilon_{0 k^{\prime}}\right) .
\end{aligned}
$$

The presence of this scattering term reflects the fact that the total energy including the spin-orbit interaction energy with the electric field is conserved in elastic collisions.

\section{B. Scattering correction due to the change in $\boldsymbol{r}$ during collisions}

We have so far ignored the presence of the term $e \boldsymbol{E} \cdot \hat{\boldsymbol{r}}$ in the time-evolution operator. At this stage we would like to 
determine the additional scattering term linear in $\boldsymbol{E}$ arising from it, which we denote by $\hat{J}_{\mathrm{sj}}^{b}\left(n_{k}\right)$. To accomplish this we use Eq. (7) to find the correction $g_{\boldsymbol{k} \boldsymbol{k}^{\prime}}^{b}$ to $g_{\boldsymbol{k} \boldsymbol{k}^{\prime}}$ arising from the presence of $e \boldsymbol{E} \cdot \hat{\boldsymbol{r}}$ in the time-evolution operator. Using Eq. (9a) we will then obtain $\hat{J}_{\mathrm{sj}}^{b}\left(n_{\boldsymbol{k}}\right)$ as $(i / \hbar)\left[\hat{H}_{U}, \hat{g}^{b}\right]_{\boldsymbol{k} \boldsymbol{k}}$.

Starting from Eq. (7), we expand the time-evolution operator to first order in the term $e \boldsymbol{E} \cdot \hat{\boldsymbol{r}}$. Using the matrix elements of the ordinary position operator $\hat{\boldsymbol{r}}$ between Bloch states

$$
\left\langle\boldsymbol{k}|\hat{\boldsymbol{r}}| \boldsymbol{k}^{\prime}\right\rangle=i \frac{\partial}{\partial \boldsymbol{k}} \delta\left(\boldsymbol{k}-\boldsymbol{k}^{\prime}\right),
$$

we obtain additional terms of the form

$$
t^{\prime} e^{-i \varepsilon_{0 \boldsymbol{k}^{t^{\prime}}}} e \boldsymbol{E} \cdot \frac{\partial}{\partial \boldsymbol{k}} \delta\left(\boldsymbol{k}-\boldsymbol{k}^{\prime}\right)=\left(\frac{i \partial}{\partial \varepsilon_{0 \boldsymbol{k}}} e^{-i \varepsilon_{0 \boldsymbol{k}^{t^{\prime}}}}\right) e \boldsymbol{E} \cdot \frac{\partial}{\partial \boldsymbol{k}} \delta\left(\boldsymbol{k}-\boldsymbol{k}^{\prime}\right) .
$$

We integrate over $t^{\prime}$ as before, and after some lengthy but straightforward algebra, we obtain

$$
\begin{aligned}
g_{\boldsymbol{k} \boldsymbol{k}^{\prime}}^{b}= & -\pi e \boldsymbol{E} \cdot \int \frac{d^{d} \boldsymbol{k}^{\prime}}{(2 \pi)^{d}}\left(\frac{\partial \bar{U}_{\boldsymbol{k} \boldsymbol{k}^{\prime}}}{\partial \boldsymbol{k}}+\frac{\partial \bar{U}_{\boldsymbol{k} \boldsymbol{k}^{\prime}}}{\partial \boldsymbol{k}^{\prime}}\right) \\
& \times\left(n_{\boldsymbol{k}}-n_{\boldsymbol{k}^{\prime}}\right) \frac{\partial}{\partial \boldsymbol{\varepsilon}_{0 \boldsymbol{k}}} \delta\left(\varepsilon_{0 \boldsymbol{k}}-\varepsilon_{0 \boldsymbol{k}^{\prime}}\right) .
\end{aligned}
$$

We have not written out explicitly a contribution to $g_{\boldsymbol{k} \boldsymbol{k}^{\prime}}^{b}$ containing terms of the form $\partial n_{\boldsymbol{k}} / \partial \boldsymbol{k}$. We find that such terms drop out in the final evaluation of spin currents when the scattering potential is elastic, as a result of integrating over $\boldsymbol{k}$ and $\boldsymbol{k}^{\prime}$. In the final analysis these terms involve the square matrix element $\left|\bar{U}_{\boldsymbol{k} \boldsymbol{k}^{\prime}}\right|^{2}$ which does not have any contributions linear in $\lambda$. We find that the leading contribution due to these terms is $\propto \lambda^{2}$ and may therefore be neglected. Evaluating the $\boldsymbol{k}$ derivatives of the impurity potentials gives

$$
\frac{\partial \bar{U}_{\boldsymbol{k} \boldsymbol{k}^{\prime}}}{\partial \boldsymbol{k}}+\frac{\partial \bar{U}_{\boldsymbol{k} \boldsymbol{k}^{\prime}}}{\partial \boldsymbol{k}^{\prime}}=-i \lambda \mathcal{U}_{\boldsymbol{k} \boldsymbol{k}^{\prime}} \boldsymbol{\sigma} \times\left(\boldsymbol{k}-\boldsymbol{k}^{\prime}\right) .
$$

Substituting this into the Eq. (18) and subsequently evaluating $\hat{J}_{\mathrm{sj}}^{b}\left(n_{k}\right)=(i / \hbar)\left[\hat{H}_{U}, \hat{g}^{b}\right]_{k k}$ we obtain the scattering term

$$
\begin{aligned}
\hat{J}_{\mathrm{sj}}^{b}\left(n_{\boldsymbol{k}}\right)= & \frac{2 \pi n_{i} e \lambda}{\hbar} \int \frac{d^{d} k^{\prime}}{(2 \pi)^{d}}\left|\mathcal{U}_{\boldsymbol{k}^{\prime} \boldsymbol{k}}\right|^{2}\left(n_{\boldsymbol{k}}-n_{\boldsymbol{k}^{\prime}}\right) \boldsymbol{E} \cdot \boldsymbol{\sigma} \\
& \times\left(\boldsymbol{k}-\boldsymbol{k}^{\prime}\right) \frac{\partial}{\partial \varepsilon_{0 \boldsymbol{k}}} \delta\left(\varepsilon_{0 \boldsymbol{k}}-\varepsilon_{0 \boldsymbol{k}^{\prime}}\right),
\end{aligned}
$$

which is easily seen to be exactly equal to $\hat{J}_{\mathrm{sj}}^{a}\left(n_{k}\right)$. The sum of these terms constitutes the total side-jump scattering term $\hat{J}_{\mathrm{sj}}\left(n_{k}\right)=\hat{J}_{\mathrm{sj}}^{a}\left(n_{k}\right)+\hat{J}_{\mathrm{sj}}^{b}\left(n_{k}\right)=2 \hat{J}_{\mathrm{sj}}^{a}\left(n_{k}\right)$, which contains the wellknown factor of 2 associated with side jump. ${ }^{37}$ We emphasize that we obtain this reinforcement of the side jump directly from the scattering term, and our work shows no evidence that it is related in any direct way to the integral of the velocity operator [see Eqs. (29) and (35) below] over the time of a collision. ${ }^{19}$

\section{CONTRIBUTION OF THE SIDE-JUMP MECHANISM TO THE SPIN HALL CURRENT}

We have derived a contribution linear in the electric field to the scattering term appearing in the kinetic equation. This contribution is brought about by the spin-dependent interaction of the charge carriers with the electric field due to the spin-orbit interaction. In this section we will first evaluate the correction that this term yields in the spin-dependent part of the density matrix and we will show that this correction accounts fully for the side-jump spin Hall current including the important factor of $2 .{ }^{19,20,37}$ This is done in Sec. IV A. To show that this is the only side-jump contribution to the spin Hall effect, Sec. IV B will demonstrate that the modifications to the velocity operator do not contribute any additional terms to the spin current.

\section{A. Contribution of the side-jump scattering term}

We need to find the contribution to the spin Hall current brought about by the additional scattering term $\hat{J}_{\mathrm{sj}}\left(n_{k}\right)$. In order to further evaluate Eq. (8) we let $f_{k}=f_{0 k}+f_{E k}$, where $f_{0 k}\left(\varepsilon_{0 k}\right)$ is the Fermi-Dirac function, which is a scalar in spin space in the case under study, and $f_{E \boldsymbol{k}}$ is the correction we will determine from the kinetic equation. First, the kineticenergy part of the Hamiltonian, $\varepsilon_{0 k}$, drops out of the commutator. Also, in the commutator $\left[\frac{1}{2} \boldsymbol{\sigma} \cdot \boldsymbol{\Delta}_{k}, f_{k}\right]$ we note that $\frac{1}{2} \boldsymbol{\sigma} \cdot \boldsymbol{\Delta}_{\boldsymbol{k}}$ is first order in the electric field, so the density matrix can be replaced with the equilibrium Fermi-Dirac function, which is a scalar in spin space, so $\left[\frac{1}{2} \boldsymbol{\sigma} \cdot \boldsymbol{\Delta}_{k}, f_{0 k}\right]=0$. Moreover, in the side-jump scattering term $\hat{J}_{\mathrm{sj}}\left(n_{k}\right)$, which is also first order in the electric field, we may replace $n_{k}$ by $f_{0 k}$. Following some short and straightforward algebra, the sidejump scattering term can be written as

$$
\hat{J}_{\mathrm{sj}}\left(f_{0 k}\right)=\frac{1}{\tau_{p}} \boldsymbol{\sigma} \cdot \boldsymbol{\Delta}_{k} \delta\left(\varepsilon-\varepsilon_{F}\right),
$$

where $\tau_{p}$ is the usual momentum relaxation time.

Next, we decompose $f_{E k}$ into a part scalar in spin space and a spin-dependent part, thus $f_{E \boldsymbol{k}}=n_{E \boldsymbol{k}} \perp+S_{E \boldsymbol{k}}$. The equation for $n_{E \boldsymbol{k}}$ in the steady state is the ordinary scalar Boltzmann equation. The term $\left[\hat{H}_{E}, \hat{f}\right]_{k}$ becomes $(e / \hbar) \boldsymbol{E} \cdot\left(\partial f_{0} / \partial \boldsymbol{k}\right)$ which is a scalar and acts as the source term for $n_{E \boldsymbol{k}}$. We write the scalar part of the Boltzmann equation as

$$
\hat{J}_{0}\left(n_{E \boldsymbol{k}}\right)=\frac{e \boldsymbol{E}}{\hbar} \cdot \frac{\partial f_{0 \boldsymbol{k}}}{\partial \boldsymbol{k}},
$$

where the scattering term $\hat{J}_{0}\left(n_{k}\right)$ has been defined in Eq. (13a). The solution for $n_{E \boldsymbol{k}}$ is written as

$$
n_{E \boldsymbol{k}}=\frac{\tau_{p} e \boldsymbol{E}}{\hbar} \cdot \frac{\partial f_{0 \boldsymbol{k}}}{\partial \boldsymbol{k}}=\frac{\hbar \tau_{p} e \boldsymbol{E} \cdot \boldsymbol{k}}{m^{*}} \frac{\partial f_{0 \boldsymbol{k}}}{\partial \varepsilon_{0 \boldsymbol{k}}} .
$$

The equation for $S_{E \boldsymbol{k}}$ in the steady state is $\hat{J}_{0}\left(S_{E \boldsymbol{k}}\right)=-\hat{J}_{\mathrm{sj}}\left(f_{0 \boldsymbol{k}}\right)$, in which the right-hand side, $\hat{J}_{\mathrm{sj}}\left(f_{0 k}\right)$, acts as a source term for $S_{E \boldsymbol{k}}$. Substituting the explicit expressions for the two scattering terms, this equation can be written in a simpler form as 


$$
\frac{S_{E k}}{\tau_{p}}=-\frac{1}{\tau_{p}} \boldsymbol{\sigma} \cdot \Delta_{k} \delta\left(\varepsilon-\varepsilon_{F}\right),
$$

and has the simple solution

$$
S_{E k}=-\boldsymbol{\sigma} \cdot \Delta_{k} \delta\left(\varepsilon-\varepsilon_{F}\right) .
$$

We would like to draw attention to the fact that the additional side-jump collision integral is part of the source for $S_{E k}$. The source term itself contains a factor of $1 / \tau_{p}$, which cancels the $1 / \tau_{p}$ appearing on the left-hand side of the equation for $S_{E k}$. This explains why the end result for the side-jump contribution to the spin Hall current does not depend on the strength and shape of the impurity potential.

Now that we have found the solution for $S_{E k}$, in other words the spin-dependent part of the density matrix in an electric field, we can determine its contribution to the spin Hall current. We denote the components of the spin velocity as $v_{j}^{i}$ which corresponds to a spin component $i$ flowing along the direction $j$. In finding the contribution to the spin Hall current due to $S_{E k}$ we can restrict ourselves to the term in the spin velocity to zeroth order in the electric field, which is $v_{j}^{i}=\left(\hbar k_{j} / m^{*}\right) \hbar \sigma_{i} / 2$. For $\boldsymbol{E}=\left(E_{x}, 0,0\right)$ the side-jump Hamiltonian $\frac{1}{2} \boldsymbol{\sigma} \cdot \boldsymbol{\Delta}_{\boldsymbol{k}}$ becomes

$$
e \lambda \boldsymbol{E} \cdot \boldsymbol{\sigma} \times \boldsymbol{k}=-e \lambda E_{x} k_{y} \sigma_{z},
$$

This gives a side-jump spin Hall current as a result of the modification to the scattering term

$$
\left.j_{y}^{z}\right|^{\text {sct }}=\left(\frac{\hbar}{2}\right) \int \frac{d^{d} k}{(2 \pi)^{d}} \frac{\hbar k_{y}}{m^{*}} \operatorname{tr}\left(\sigma_{z} S_{E k}\right)=n e \lambda E_{x},
$$

where the trace is taken over the spin components and $n$ is the density. This term therefore gives a spin Hall conductivity $\left.\sigma_{y x}^{z}\right|^{\mid s c t}=n e \lambda$, which is the usual side-jump term in the spin Hall current. ${ }^{19,20,23,25,37,45}$ This result is valid in both two and three dimensions.

\section{B. Vanishing contribution of the corrections to the velocity operator}

It will be shown in Sec. IV B that the correction to the velocity operator linear in the electric field does not yield any additional terms in the spin Hall current. The velocity operator is defined as the time derivative of the physical position operator, which in turn is given by

$$
\hat{\boldsymbol{r}}_{\mathrm{phys}}=\hat{\boldsymbol{r}}+\lambda \hat{\boldsymbol{\sigma}} \times \hat{\boldsymbol{k}} .
$$

Notice that all spin-orbit interactions can be most directly derived by replacing $\boldsymbol{r}$ by $\boldsymbol{r}_{\text {phys }}$ in the direct interactions and expanding to first order in $\lambda$. The requirement $\lambda k_{F}^{2} \ll 1$ noticed above implies that the corrections emerging from the second term in Eq. (28) will be small as compared with the first term.

The velocity operator has a part which is diagonal in $\boldsymbol{k}$ and is given by

$$
\begin{aligned}
\boldsymbol{v}_{\boldsymbol{k}} & =(i / \hbar)\left[\hat{H}, \hat{\boldsymbol{r}}_{\mathrm{phys}}\right]_{k} \\
& =\frac{\hbar \boldsymbol{k}}{m^{*}}+\frac{i}{\hbar}[e \lambda \boldsymbol{E} \cdot \hat{\boldsymbol{\sigma}} \times \hat{\boldsymbol{k}}, \hat{\boldsymbol{r}}]_{k}+\frac{i}{\hbar}[e \boldsymbol{E} \cdot \hat{\boldsymbol{r}}, \lambda \hat{\boldsymbol{\sigma}} \times \hat{\boldsymbol{k}}]_{k} \\
& =\frac{\hbar \boldsymbol{k}}{m^{*}}-\frac{2 e \lambda}{\hbar} \boldsymbol{\sigma} \times \boldsymbol{E} .
\end{aligned}
$$

The $\boldsymbol{k}$-diagonal part of the spin velocity $v_{j}^{i}$, up to first order in the electric field, is thus

$$
v_{j}^{i}=\frac{\hbar k_{j}}{m^{*}} \frac{\hbar \sigma_{i}}{2}-\frac{e \lambda}{2}\left\{(\boldsymbol{\sigma} \times \boldsymbol{E})_{j}, \sigma_{i}\right\},
$$

where $\{A, B\} \equiv A B+B A$. For an electric field along $\hat{\boldsymbol{x}}$ we obtain for the $\boldsymbol{k}$-diagonal part of the spin velocity $v_{y}^{z}$ the expression

$$
v_{y}^{z}=\frac{\hbar}{2} \frac{\hbar k_{y}}{m^{*}} \sigma_{z}-e \lambda E_{x} .
$$

The $\boldsymbol{E}$-dependent part of the spin velocity operator is a scalar in spin space and its contribution to the spin Hall current is found by multiplying by the scalar part of the equilibrium density matrix $f_{0 k}$. It gives us the term

$$
\left.j_{y}^{z}\right|^{v e l, d}=-e \lambda E_{x} \int \frac{d^{d} k}{(2 \pi)^{d}} \operatorname{tr} f_{0 k}=-n e \lambda E_{x}
$$

so its contribution to the spin Hall conductivity is $\left.\sigma_{y x}^{z}\right|^{\text {vel, } d}$ $=-n e \lambda$.

This is, however, not the full story. The velocity operator also has a term that is off-diagonal in the wave vector, which is referred to as $\boldsymbol{v}_{\boldsymbol{k} \boldsymbol{k}^{\prime}}$ and is given by

$$
\boldsymbol{v}_{\boldsymbol{k} \boldsymbol{k}^{\prime}}=\frac{i}{\hbar}\left[\hat{H}_{U}, \hat{\boldsymbol{r}}_{\mathrm{phys}}\right]_{\boldsymbol{k} k^{\prime}} .
$$

The matrix elements of the impurity potential are given by Eq. (5). The part of the matrix element $\boldsymbol{v}_{\boldsymbol{k} \boldsymbol{k}^{\prime}}$ originating from $\hat{\boldsymbol{r}}$ is easily seen to be

$$
\frac{i}{\hbar}[\hat{U}, \hat{\boldsymbol{r}}]_{k k^{\prime}}=\frac{1}{\hbar}\left(\frac{\partial U_{\boldsymbol{k} \boldsymbol{k}^{\prime}}}{\partial \boldsymbol{k}^{\prime}}+\frac{\partial U_{\boldsymbol{k} \boldsymbol{k}^{\prime}}}{\partial \boldsymbol{k}}\right) .
$$

The disorder potential is the potential $\hat{U}$ due to the full ensemble of impurities present in the system. In the final result for the spin Hall current, the $\boldsymbol{k}$-off-diagonal part of the velocity operator will be traced with the $\boldsymbol{k}$-off-diagonal part of the density matrix $g_{k k^{\prime}}$, which in the first Born approximation is also linear in $\hat{U}$. Once this is done, a configurational average will be performed over the impurities. In the end we seek the result to first order in $\lambda$. However, it proves more straightforward to work in terms of the full potential $\hat{U}$ until the end. Only then we will restrict ourselves to the terms which are first order in $\lambda$.

With these insights in mind we proceed, Eq. (33) yields

$$
\boldsymbol{v}_{k k^{\prime}}=-\frac{2 i \lambda}{\hbar} \boldsymbol{\sigma} \times\left(\boldsymbol{k}-\boldsymbol{k}^{\prime}\right) U_{\boldsymbol{k} k^{\prime}},
$$

where we have written the matrix elements of the full 
potential $\hat{U}$. Note that this expression has not been averaged over impurity configurations. This expression for the $\boldsymbol{k}$-off-diagonal part of the velocity operator holds because, for elastic scattering, the scalar part of the scattering potential $\mathcal{U}_{\boldsymbol{k} \boldsymbol{k}^{\prime}} \equiv \mathcal{U}\left(\boldsymbol{k}-\boldsymbol{k}^{\prime}\right)$ depends only on the difference $\boldsymbol{k}-\boldsymbol{k}^{\prime}$. Its contribution to the velocity operator is immediately seen to be zero. This can also be understood by noting that the scalar part of the impurity potential commutes with $\boldsymbol{r}$ and does not contribute to the velocity.

The $\boldsymbol{k}$-off-diagonal part of the velocity operator contributes to the side-jump spin Hall current. To find its contribution we return to Eq. (7) and integrate over time to find

$$
g_{k k^{\prime}}=i \pi \delta\left(\varepsilon_{0 k}-\varepsilon_{0 k^{\prime}}\right) U_{k k^{\prime}}\left(f_{k}-f_{k^{\prime}}\right) .
$$

This expression also contains the matrix elements of the full impurity potential and has not been averaged over impurity configurations.

The contribution of the $\boldsymbol{k}$-off-diagonal part of the velocity operator to the spin current is found by taking the trace of the spin velocity arising from Eq. (35) with the $\boldsymbol{k}$-off-diagonal part of the density matrix given in Eq. (36). This yields for the spin Hall current

$$
\begin{aligned}
j_{y}^{z} \mid \text { vel,od }= & \frac{\hbar}{2} \operatorname{tr} \sigma_{z} \int \frac{d^{d} k}{(2 \pi)^{d}} \int \frac{d^{d} k^{\prime}}{(2 \pi)^{d}} v_{\boldsymbol{k} \boldsymbol{k}^{\prime}}^{y} g_{\boldsymbol{k}^{\prime} \boldsymbol{k}} \\
= & -(\lambda \hbar) \frac{2 \pi}{\hbar} \int \frac{d^{d} k}{(2 \pi)^{d}}\left(k_{x}-k_{x}^{\prime}\right)\left\langle U_{\boldsymbol{k} \boldsymbol{k}^{\prime}} U_{\boldsymbol{k}^{\prime} \boldsymbol{k}}\right\rangle \\
& \times \delta\left(\varepsilon_{0 \boldsymbol{k}^{\prime}}-\varepsilon_{0 k}\right)\left(f_{\boldsymbol{k}}-f_{\boldsymbol{k}^{\prime}}\right),
\end{aligned}
$$

where the bracket denotes the average over impurity configurations. At this stage we introduce the simplification that we require only terms to first order in $\lambda$. We note that, since the entire term in Eq. (37) already contains $\lambda$, the other terms in this equation are needed only to zeroth order in $\lambda$. Consider first the term proportional to $k_{x}$, which, to first order in $\lambda$, can be written as

$$
\begin{aligned}
& -(\lambda \hbar) \int \frac{d^{d} k}{(2 \pi)^{d}} k_{x}\left[\frac{2 \pi}{\hbar} \int \frac{d^{d} k^{\prime}}{(2 \pi)^{d}}\left\langle U_{k k^{\prime}} U_{\boldsymbol{k}^{\prime} k}\right\rangle\right. \\
& \left.\times \delta\left(\varepsilon_{0 \boldsymbol{k}^{\prime}}-\varepsilon_{0 k}\right)\left(n_{\boldsymbol{k}}-n_{\boldsymbol{k}^{\prime}}\right)\right] \\
& =-(\lambda \hbar) \int \frac{d^{d} k}{(2 \pi)^{d}} k_{x} \hat{J}_{0}\left(n_{\boldsymbol{k}}\right) \\
& =-(\lambda \hbar) \int \frac{d^{d} k}{(2 \pi)^{d}} k_{x}\left(\frac{e E_{x}}{\hbar} \frac{\partial f_{0 k}}{\partial k_{x}}\right),
\end{aligned}
$$

where the last replacement follows from the scalar Boltzmann equation, as written in Eq. (22), assuming, as before, that $\boldsymbol{E} \| \hat{\boldsymbol{x}}$. Further, Eq. (37) also contains a term proportional to $k_{x}^{\prime}$, which is easily seen to give exactly the same contribution if one swaps $\boldsymbol{k}$ and $\boldsymbol{k}^{\prime}$ in the summation. The contribution of the $\boldsymbol{k}$-off-diagonal part of the velocity operator to the spin Hall current to first order in $\lambda$ is therefore

$$
j_{y}^{z} \mid \text { vel,od }=-2 \lambda e E_{x} \int \frac{d^{d} k}{(2 \pi)^{d}} k_{x} \frac{\partial f_{0 k}}{\partial k_{x}}=n e \lambda E_{x} .
$$

The spin Hall conductivity originating from this term is $\sigma_{y x}^{z}{ }^{\mid v e l, o d}=n e \lambda$ and it exactly cancels the contribution $\left.\sigma_{y x}^{z}\right|^{v e l, d}$ from the $\boldsymbol{k}$-diagonal $\boldsymbol{E}$-dependent part of the velocity operator. The physical explanation of this cancellation is the following. Notice that the full spin-dependent part of the velocity operator, from Eqs. (29) and (35) is

$$
-\frac{2 e \lambda}{\hbar} \boldsymbol{\sigma} \times \boldsymbol{E}-\frac{2 i \lambda}{\hbar} \boldsymbol{\sigma} \times\left(\boldsymbol{k}-\boldsymbol{k}^{\prime}\right) \mathcal{U}_{\boldsymbol{k} \boldsymbol{k}^{\prime}}
$$

which contains the total force acting on the system. According to Ehrenfest's theorem, the expectation values of position and momentum obey time-evolution equations analogous to those of classical mechanics. Consequently the expectation value of the force should be zero in the steady state, consistent with the earlier suggestion that the total force acting on the system does not contribute to the spin current. ${ }^{23}$ We note also that the presence of the velocity terms off-diagonal in wave vector is crucial in obtaining the correct side-jump contribution in the diagrammatic Kubo-formula approach, as demonstrated in Ref. 20.

\section{SUMMARY AND DISCUSSION}

We have completed the formal derivation of the side-jump spin Hall current, where we have considered (in the absence of intrinsic spin precession) all contributions to the kinetic equation in the first Born approximation. We will now discuss our findings and their implications. It is evident from our analysis that, within the kinetic equation framework, the side-jump spin Hall current originates solely from the modification of the Boltzmann collision integral due to the spindependent interaction energy of an electron with the external electric field and the impurity field. The density-matrix formulation of the problem shows that the spin-orbit interaction with the electric field alters the condition for energy conservation since the total energy conserved during collisions must include the spin-dependent part. In addition, the spinorbit coupling with the impurities causes a change in the position of the electron during scattering process, which again affects the energy balance via the interaction energy $e \boldsymbol{E} \cdot \boldsymbol{r}$. This effect doubles the size of the side-jump current.

The understanding of the side-jump effect emerging from this derivation differs from the conventional explanation, according to which this phenomenon is attributed to the linear-in- $\boldsymbol{E}$ modification of the velocity operator. It is clearly seen in the previous section that the full velocity operator in the presence of spin-orbit interactions contains an extra term due to the impurity potential, which is off-diagonal in wave vector. This term, and the $\boldsymbol{k}$-diagonal velocity operator result in two corrections to the spin current that are equal in magnitude but opposite sign so they cancel out. This could be justified informally using the fact that the velocity operator contains the total force acting on the system (or rather the term to leading order in $\lambda$ of this force), and therefore should vanish in the steady state. ${ }^{23}$ Therefore, contrary to conventional assumptions, the side-jump contribution to the spin 
Hall current is traced to the qualitatively different carrier spin dynamics during collisions. It is not traced to any linear-in- $\boldsymbol{E}$ correction to the velocity operator. The linear-in- $\boldsymbol{E}$ correction to the velocity operator is canceled by the contribution of the off-diagonal in $\boldsymbol{k}$ correction to the velocity operator. The importance of this off-diagonal-in- $\boldsymbol{k}$ correction in the velocity operator in obtaining the correct side-jump current was recognized in the diagrammatic Kuboformula approach as shown in Ref. 20.

We emphasize that in obtaining our results we have used a rigorous quantum mechanical formulation, starting with the quantum Liouville equation and making, in the course of the derivation, the same assumptions that are characteristically made in linear-response theory. Therefore our formalism could in some sense be regarded as being built from the ground up. The approach we have used demonstrates that the derivation of the side jump does not need to rely on intuitive semiclassical ideas as long as the collision integral is derived rigorously from the fundamental starting point of all transport theories. In contrast, Ref. 37 using semiclassical Boltzmann arguments counted six terms contributing to side jump, but did not clearly indicate which terms should cancel. Since for the conduction band the side-jump contributions have the same magnitude but opposite signs, it led to freedom in choosing which terms cancel and still obtaining the correct amplitude of the side jump. For example, Ref. 19 counted the terms from the anomalous velocity and the shift of the position operator. Our analysis shows exactly which terms are nonzero and which contributions cancel and is in agreement with the Kubo derivation of side-jump contributions presented in Refs. 20 and 49. Our results are also in agreement with recent extensive studies of the anomalous Hall effect in magnetic semiconductors. ${ }^{42,44,51}$ In particular, Refs. 42 and 52, by comparing the semiclassical description of the side jump with the Kubo and Keldysh formalisms, found that the velocity operator is unchanged and that the two contributions to the side jump have different origins due to the renormalization of the distribution function and due to the modification of the conservation of energy.

\section{ACKNOWLEDGMENTS}

We would like to acknowledge stimulating discussions with S. Das Sarma, Jairo Sinova, Hans-Andreas Engel and Peter Schwab. D.C. was supported by LPS-NSA-CMTC. E.M.H. was financially supported by DFG under Grant No. HA 5893/1-1. Work at Argonne was supported by DOE BES under Contract No. DE-AC02-06CH11357. G.V. acknowledges support from NSF under Grant No. DMR-0705460.
${ }^{1}$ I. Žutić, J. Fabian, and S. Das Sarma, Rev. Mod. Phys. 76, 323 (2004).

${ }^{2}$ D. D. Awschalom and M. E. Flatté, Nat. Phys. 3, 153 (2007).

${ }^{3}$ M. I. Dyakonov and V. I. Perel, Phys. Lett. A 35, 459 (1971).

${ }^{4}$ J. E. Hirsch, Phys. Rev. Lett. 83, 1834 (1999).

${ }^{5}$ S. Zhang, Phys. Rev. Lett. 85, 393 (2000).

${ }^{6}$ S. Murakami, N. Nagaosa, and S.-C. Zhang, Science 301, 1348 (2003).

${ }^{7}$ J. Sinova, D. Culcer, Q. Niu, N. A. Sinitsyn, T. Jungwirth, and A. H. MacDonald, Phys. Rev. Lett. 92, 126603 (2004).

${ }^{8}$ H.-A. Engel, E. I. Rashba, and B. I. Halperin, in Handbook of Magnetism and Advanced Magnetic Materials, edited by $\mathrm{H}$. Kronmüller and S. Parkin (Wiley, Chichester, UK, 2007), Vol. V, pp. 2858-2877.

${ }^{9}$ R. Winkler, in Handbook of Magnetism and Advanced Magnetic Materials, edited by H. Kronmüller and S. Parkin (Wiley, Chichester, UK, 2007), Vol. V, pp. 2830-2843.

${ }^{10}$ Y. K. Kato, R. C. Myers, A. C. Gossard, and D. D. Awschalom, Science 306, 1910 (2004).

${ }^{11}$ V. Sih, R. C. Myers, Y. K. Kato, W. H. Lau, A. C. Gossard, and D. D. Awschalom, Nat. Phys. 1, 31 (2005).

${ }^{12}$ J. Wunderlich, B. Kaestner, J. Sinova, and T. Jungwirth, Phys. Rev. Lett. 94, 047204 (2005)

${ }^{13}$ N. P. Stern, D. W. Steuerman, S. Mack, A. C. Gossard, and D. D. Awschalom, Nat. Phys. 4, 843 (2008).

${ }^{14}$ D. Culcer and R. Winkler, Phys. Rev. B 76, 245322 (2007).

${ }^{15}$ W.-K. Tse, J. Fabian, I. Žutić, and S. Das Sarma, Phys. Rev. B 72, 241303(R) (2005)

${ }^{16}$ V. M. Galitski, A. A. Burkov, and S. Das Sarma, Phys. Rev. B 74, 115331 (2006).
${ }^{17}$ N. P. Stern, S. Ghosh, G. Xiang, M. Zhu, N. Samarth, and D. D. Awschalom, Phys. Rev. Lett. 97, 126603 (2006).

${ }^{18}$ C. Brüene, A. Roth, E. Novik, M. Köenig, H. Buhmann, E. Hankiewicz, W. Hanke, J. Sinova, and L. Molenkamp, arXiv:0812.3768 (unpublished).

${ }^{19}$ H. A. Engel, B. I. Halperin, and E. I. Rashba, Phys. Rev. Lett. 95, 166605 (2005).

${ }^{20}$ W. K. Tse and S. Das Sarma, Phys. Rev. Lett. 96, 056601 (2006).

${ }^{21}$ A. Crépieux and P. Bruno, Phys. Rev. B 64, 014416 (2001).

${ }^{22}$ V. K. Dugaev, P. Bruno, M. Taillefumier, B. Canals, and C. Lacroix, Phys. Rev. B 71, 224423 (2005).

${ }^{23}$ E. M. Hankiewicz and G. Vignale, Phys. Rev. B 73, 115339 (2006).

${ }^{24}$ J. L. Cheng and M. W. Wu, J. Phys.: Condens. Matter 20, 085209 (2008).

${ }^{25}$ E. M. Hankiewicz and G. Vignale, J. Phys.: Condens. Matter 21, 253202 (2009).

${ }^{26}$ J. I. Inoue, G. E. W. Bauer, and L. W. Molenkamp, Phys. Rev. B 70, 041303(R) (2004).

${ }^{27}$ P. L. Krotkov and S. Das Sarma, Phys. Rev. B 73, 195307 (2006).

${ }^{28}$ E. G. Mishchenko, A. V. Shytov, and B. I. Halperin, Phys. Rev. Lett. 93, 226602 (2004).

${ }^{29}$ R. Karplus and J. M. Luttinger, Phys. Rev. 95, 1154 (1954).

${ }^{30}$ J. M. Luttinger, Phys. Rev. 112, 739 (1958).

${ }^{31}$ J. Smit, Physica (Amsterdam) 21, 877 (1955).

${ }^{32}$ J. Smit, Physica (Amsterdam) 24, 39 (1958).

${ }^{33}$ W. Kohn and J. M. Luttinger, Phys. Rev. 108, 590 (1957).

${ }^{34}$ L. Berger, Phys. Rev. B 2, 4559 (1970). 
${ }^{35}$ L. Berger, Phys. Rev. B 5, 1862 (1972).

${ }^{36}$ C. Lewiner, O. Betbeder-Matibet, and P. Nozières, J. Phys. Chem. Solids 34, 765 (1973).

${ }^{37}$ P. Nozières and C. Lewiner, J. Phys. (Paris) 34, 901 (1973).

${ }^{38}$ T. Jungwirth, Q. Niu, and A. H. MacDonald, Phys. Rev. Lett. 88, 207208 (2002).

${ }^{39}$ Y. Yao, L. Kleinman, A. H. MacDonald, J. Sinova, T. Jungwirth, D.-S. Wang, E. Wang, and Q. Niu, Phys. Rev. Lett. 92, 037204 (2004).

${ }^{40}$ S. Onoda, N. Sugimoto, and N. Nagaosa, Prog. Theor. Phys. 116, 61 (2006)

${ }^{41}$ T. S. Nunner et al., Phys. Rev. B 76, 235312 (2007).

${ }^{42}$ N. A. Sinitsyn, A. H. MacDonald, T. Jungwirth, V. K. Dugaev, and J. Sinova, Phys. Rev. B 75, 045315 (2007).

${ }^{43}$ N. A. Sinitsyn, J. Phys.: Condens. Matter 20, 023201 (2008).
${ }^{44}$ N. Nagaosa, J. Sinova, S. Onoda, A. MacDonald, and N. Ong, arXiv:0904.4154 (unpublished).

${ }^{45}$ W. K. Tse and S. Das Sarma, Phys. Rev. B 74, 245309 (2006).

${ }^{46}$ L. Hu, Z. Huang, and S. Hu, Phys. Rev. B 73, 235314 (2006).

${ }^{47}$ E. M. Hankiewicz and G. Vignale, Phys. Rev. Lett. 100, 026602 (2008).

${ }^{48}$ R. Raimondi and P. Schwab, EPL 87, 37008 (2009).

${ }^{49}$ E. M. Hankiewicz, G. Vignale, and M. E. Flatté, Phys. Rev. Lett. 97, 266601 (2006).

${ }^{50}$ J. Rammer, Quantum Transport Theory (Westview, Reading, 2004).

${ }^{51}$ A. A. Kovalev, Y. Tserkovnyak, K. Výborný, and J. Sinova, Phys. Rev. B 79, 195129 (2009).

${ }^{52}$ N. A. Sinitsyn, Q. Niu, J. Sinova, and K. Nomura, Phys. Rev. B 72, 045346 (2005). 